\title{
ON DIFFERENTIATION OF INTEGRALS AND APPROXIMATE CONTINUITY
}

\author{
ARTHUR ROSENTHAL
}

The following discussion is closely connected with Lebesgue's theorem that the derivative of an integral is equal to the integrand almost everywhere. It is well known that in generalizing this theorem to higher dimensions, great care must be exercised in the choice of the systems of intervals or sets used for $n$-dimensional differentiation.

Lebesgue $^{1}$ had already observed that arbitrary intervals (parallel to the axes) cannot be used for the generalization of that theorem, but only such intervals whose edges have a bounded ratio, or, more generally, such sets which are regular relative to the cubes. This also corresponds to the behavior of the most essential tool used in the proof, namely, Vitali's covering theorem. Saks ${ }^{2}$ and, independently, Busemann and Feller ${ }^{3}$ found later that there is a remarkable difference between the integrals of bounded ${ }^{4}$ and unbounded functions: in the first (but not generally in the last) case, differentiation relative to arbitrary intervals (parallel to the axes) furnishes the integrand almost everywhere. But, according to Zygmund and Nikodym ${ }^{5}$ and to Busemann and Feller, ${ }^{3}$ even in the case of bounded integrands, differentiation relative to the system of all rectangular parallelopipeds (arbitrarily oriented) does not always furnish the integrand almost everywhere.

As to integrals in abstract spaces-in the case of bounded integrands, de Possel $^{6}$ gave necessary and sufficient conditions for the systems of sets used in differentiation to permit a generalization of Lebesgue's theorem; while in case of arbitrary integrands, de Possel ${ }^{6}$

Presented to the Society, September 5, 1941; received by the editors July 14, 1941.

${ }^{1}$ H. Lebesgue, Annales de l'École Normale, (3), vol. 27 (1910), pp. 363, 387.

${ }^{2} \mathrm{~S}$. Saks, Théorie de l'Intégrale, Warsaw, 1933, p. 232; Theory of the Integral, Warsaw, Lwow, 1937, p. 132.

${ }^{3}$ H. Busemann and W. Feller, Fundamenta Mathematicae, vol. 22 (1934), pp. 226-256.

${ }^{4}$ Further generalizations: A. Zygmund, Fundamenta Mathematicae, vol. 23 (1934), pp. 143-149; B. Jessen, J. Marcinkiewicz, A. Zygmund, Fundamenta Mathematicae, vol. 25 (1935), pp. 217-234.

${ }^{5}$ O. Nikodym, Fundamenta Mathematicae, vol. 10 (1927), pp. 167-168 (note cf. A. Zygmund).

${ }^{6}$ R. de Possel, Comptes Rendus de l'Académie des Sciences, Paris, vol. 201 (1935), pp. 579-581; Journal de Mathématiques, (9), vol. 15 (1936), pp. 391-409. 
and Hahn (in a still unpublished manuscript ${ }^{7}$ ) gave sufficient conditions suggested by Vitali's covering theorem.

On the other hand, in the case of bounded integrands, the original result of Lebesgue has been supplemented in an essential way by a theorem of Denjoy, ${ }^{8}$ namely, that differentiation of an integral furnishes the integrand certainly wherever the integrand is approximately continuous. Denjoy proved this theorem for the case of one dimension, Saks ${ }^{2}$ generalized it for more dimensions, namely-as in Lebesgue's theorem for bounded functions - using arbitrary intervals. Of course, the definition of approximate continuity depends on the notion of the density of a set $M$, and as the density of $M$ results from differentiation of the characteristic function of $M$, the notion of approximate continuity also depends on the systems of sets used for this differentiation. Therefore, it is natural to use the same systems for definition of density and consequently of approximate continuity as for differentiation of the integral, especially if we are speaking (as in the theorem of Denjoy) about approximate continuity and differentiation simultaneously.

In preparing the second volume of Hahn's Reelle Funktionen, ${ }^{7}$ I observed that there is an essential difference between the generalization of Lebesgue's theorem and that of Denjoy: While in the case of Lebesgue's theorem (also for bounded integrands) only rather special systems of sets can be used for differentiation, it is possible for the generalization of Denjoy's theorem, in any metric space (as will be shown), to differentiate relative to quite arbitrary, only indefinitely fine, systems of sets (see Theorem 1 ). ${ }^{9}$ In a certain sense (namely, for regular derivatives), the approximate continuity at a point is even necessary and sufficient for the derivative of the integral to be equal to the integrand, assumed as bounded (see Theorem 4).

Another theorem of Denjoy ${ }^{10}$ states that every measurable function is approximately continuous almost everywhere. The systems of sets

${ }^{7}$ This manuscript forms the basis of the second volume of Hahn's Reelle Funktionen which is now being elaborated and prepared for publication by the author of the present paper. The more general systems used by de Possel (see Footnote 6) in the case of bounded integrands, or the quite arbitrary "indefinitely fine" systems to be used here, had not been considered by Hahn. The notations of this second volume are used here.

${ }^{8}$ A. Denjoy, Sur les fonctions dérivées sommables, Bulletin de la Société Mathématique de France, vol. 43 (1915), p. 172.

${ }^{9}$ For instance, in the $n$-space relative to the system of all the rectangular parallelopipeds (arbitrarily oriented).

${ }_{10}$ A. Denjoy, loc. cit., p. 170; see also W. Sierpinski, Fundamenta Mathematicae, vol. 3 (1922), p. 320 . 
used in the generalization of this theorem can be the same ${ }^{11}$ as, but cannot be more general than, those used in the generalization of Lebesgue's theorem for bounded integrands, for the two theorems of Denjoy taken together immediately imply Lebesgue's theorem for bounded integrands.

Thus we have the following situation: For the generalization of Denjoy's theorem that the derivative of an integral furnishes the integrand (assumed as bounded) certainly wherever the integrand is approximately continuous, the systems of sets used for differentiation can be chosen quite arbitrarily; yet in order to get Lebesgue's result that the integrand is obtained almost everywhere (or Denjoy's second result that the integrand is approximately continuous almost everywhere), the systems of sets employed must be rather special.

Let $R$ be a metric space, $\mathfrak{M}$ a $\sigma$-field consisting of subsets of $R$, with $R \in \mathfrak{M}$; let $\phi$ and $\psi$ be totally additive, finite set functions in $\mathfrak{M}$; let $\psi(M) \geqq 0$ for $M \in \mathfrak{M}$, (so that $\psi(M)$ is monotone increasing in $\mathfrak{M}$ ), and let $\mathfrak{M}$ be complete for $\psi$.

A system $\mathfrak{Q} \subseteq \mathfrak{M}$ of sets may be called an indefinitely fine system of sets, if to every point $a \in R$ there corresponds a certain subsystem $\mathfrak{Q}_{a} \subseteq \mathfrak{Q}$ consisting of non-empty sets such that for every $\rho>0$ there is a set $Q \in \mathfrak{Q}_{a}$ contained in the sphere $S_{a \rho}$ (with center $a$ and radius $\rho$ ).

If there is a sequence of sets $Q_{\nu} \in \mathfrak{Q}_{a}$ converging to $a$ so that the sequence ${ }^{12} \phi\left(Q_{v}\right) / \psi\left(Q_{\nu}\right)$ converges to a limit $d,{ }^{13}$ then $d$ will be called a derivate of $\phi$ with respect to $\psi$ at the point $a$ relative to $\mathfrak{Q}$. The largest and smallest of these numbers $d^{13}$ (if $a$ is fixed) are called the upper and lower derivate of $\phi$ with respect to $\psi$ at $a$ relative to $\mathfrak{Q}$ :

$$
\bar{D}(a, \phi, \psi, \mathfrak{Q}), \quad \underline{D}(a, \phi, \psi, \mathfrak{Q}) .
$$

If

$$
\bar{D}(a, \phi, \psi, \mathfrak{Q})=\underline{D}(a, \phi, \psi, \mathfrak{\Omega}),
$$

we call this value the derivative $D(a, \phi, \psi, \mathfrak{Q})$ of $\phi$ with respect to $\psi$ at $a$ relative to $\mathfrak{Q}$.

Following Lebesgue ${ }^{1}$ we say that a sequence of sets $M_{\nu} \in \mathfrak{M}$ converges regularly to $a$ (for $\psi$ relative to $\mathfrak{Q}$ ), if there exists a number $\zeta(a)>0$ and a sequence of sets $Q_{\nu} \in \mathfrak{Q}_{a}$ converging to $a$, such that for almost all $\nu$ we have:

\footnotetext{
${ }^{11}$ We shall not prove it here.

${ }^{12}$ If $\psi\left(Q_{\nu}\right)=0$, this quotient is to be $0,+\infty,-\infty$, according as $\phi\left(Q_{v}\right)=0,>0,<0$.

${ }^{13}$ Which may be finite or infinite.
} 


$$
M_{\nu} \subseteq Q_{\nu}, \quad \psi\left(M_{\nu}\right) \geqq \zeta \cdot \psi\left(Q_{\nu}\right) .
$$

If we have $\phi\left(M_{\nu}\right) / \psi\left(M_{\nu}\right) \rightarrow D(a, \phi, \psi, \mathfrak{Q})$ for every sequence $M_{\nu}$ converging regularly to $a$ (for $\psi$ relative to $\mathfrak{\Omega}$ ), we say that the derivative $D(a, \phi, \psi, \mathfrak{Q})$ is regular at $a$.

If $A \in \mathfrak{M}$, let us set $\phi(M)=\psi(A M)$; then $\bar{D}(a, \phi, \psi, \mathfrak{Q}), D(a, \phi, \psi, \mathfrak{\Omega})$, and $D(a, \phi, \psi, \mathfrak{Q})$ become the upper density $\bar{d}(a, A, \psi, \mathfrak{Q})$, the lower density $\underline{d}(a, A, \psi, \mathfrak{Q})$, and the density $d(a, A, \psi, \mathfrak{Q})$ of $A$ at $a$ for $\psi$ relative to $\mathfrak{Q}$; all these values are greater than or equal to 0 and less then or equal to 1 .

Let $R^{*}$ be the set of all those $a \in R$ for which there is a sequence of sets $Q_{\nu} \in \mathfrak{Q}_{a}$ converging to $a$, such that $\psi\left(Q_{\nu}\right)=0$. Then we have:

$$
\underline{d}(a, A, \psi, \mathfrak{Q})=0,
$$

for all $a \in R^{*}$.

Lemma 1. For every $a \in R-R^{*}$ we have:

$$
\begin{aligned}
\underline{d}(a, A, \psi, \mathfrak{Q})+\bar{d}(a, R-A, \psi, \mathfrak{Q}) & =1 ; \\
\bar{d}(a, A, \psi, \mathfrak{Q})+\underline{d}(a, R-A, \psi, \mathfrak{Q}) & =1 .
\end{aligned}
$$

Proof. If $Q \in \mathfrak{Q}_{a}$, then $\psi(A Q)+\psi((R-A) Q)=\psi(Q)$; thus, if $\psi(Q) \neq 0: \psi(A Q) / \psi(Q)+\psi((R-A) Q) / \psi(Q)=1$.

Lemma 2. If $d\left(a, A_{1}, \psi, \mathfrak{Q}\right)=0$ and $d\left(a, A_{2}, \psi, \mathfrak{Q}\right)=0$, then also $d\left(a, A_{1}+A_{2}, \psi, \mathfrak{Q}\right)=0$.

Proof. Let $Q_{\nu} \in \mathfrak{Q}_{a}$ be a sequence converging to $a$ and choose $\epsilon>0$; then $\psi\left(A_{1} Q_{v}\right) \leqq \epsilon \psi\left(Q_{\nu}\right), \psi\left(A_{2} Q_{v}\right) \leqq \epsilon \psi\left(Q_{\nu}\right)$ for almost all $\nu$; thus also $\psi\left(\left(A_{1}+A_{2}\right) Q_{v}\right) \leqq 2 \epsilon \psi\left(Q_{v}\right)$, that is, $\psi\left(\left(A_{1}+A_{2}\right) Q_{v}\right) / \psi\left(Q_{v}\right) \rightarrow 0$.

Lemma 3. If $d\left(a, A_{1}, \psi, \mathfrak{Q}\right)=1$ and $d\left(a, A_{2}, \psi, \mathfrak{Q}\right)=1$, then also $d\left(a, A_{1} A_{2}, \psi, \Omega\right)=1$.

Proof. According to (2): $a \in R-R^{*}$; therefore because of Lemma 1, $d\left(a, R-A_{1}, \psi, \mathfrak{Q}\right)=0, d\left(a, R-A_{2}, \psi, \mathfrak{Q}\right)=0$, which implies by Lemma 2, $d\left(a, R-A_{1} A_{2}, \psi, \mathfrak{Q}\right)=0$; therefore again because of Lemma 1 , $d\left(a, A_{1} A_{2}, \psi, \mathfrak{Q}\right)=1$.

If $\alpha, \beta$ are two real numbers ( $\pm \infty$ permitted), we write: ${ }^{14}$ $\| \alpha-\beta||=|S(\alpha)-S(\beta)|$, where $S(\xi)$ means the "bounding transformation" $\xi /(1+|\xi|)$. Furthermore, $[f(x)<y]$ denotes the set of all $x \in R$, for which $f(x)<y$ holds; similar notations are used in analogous cases.

In the following, let $f$ always be a $\psi$-measurable function (that is, measurable with respect to $\psi$ as measure).

\footnotetext{
${ }^{14}$ H. Hahn, Reelle Funktionen, I, Leipzig, 1932, p. 178.
} 
The function $f$ is called approximately continuous ${ }^{15}$ at the point $a$ (for $\psi$ relative to $\mathfrak{D}$ ), if for every $\epsilon>0$ the set $[\|f(x)-f(a)\|<\epsilon]$ has density 1 at $a$ (for $\psi$ relative to $\mathfrak{Q}$ ). If $f(a)$ is finite, in this definition $\|f(x)-f(a)\|<\epsilon$ may be replaced by $|f(x)-f(a)|<\epsilon$.

Equation (2) immediately implies the following lemma.

LEMMA 4. If $f$ is approximately continuous at a (for $\psi$ relative to $\mathfrak{Q})$, then $a \in R-R^{*}$.

LEMMA 5.16 In order that $f$ be approximately continuous at a (for $\psi$ relative to $\mathfrak{Q})$, it is necessary and sufficient that for every $y>f(a)$ and for every $y^{\prime}<f(a)$ the sets $[f(x)<y]$ and $\left[f(x)>y^{\prime}\right]$ have density 1 at a ( for $\psi$ relative to $\mathfrak{D}$ ).

Necessity. Let $\epsilon>0$ be sufficiently small; then $y>f(a), y^{\prime}<f(a)$ imply $[\|f(x)-f(a)\|<\epsilon] \subseteq[f(x)<y]$ and $\subseteq\left[f(x)>y^{\prime}\right]$.

Sufficiency. If for every $y>f(a)$ and every $y^{\prime}<f(a)$ the sets $[f(x)<y]$ and $\left[f(x)>y^{\prime}\right]$ have density 1 at $a$, then, because of Lemma 3 , the same is true for the set $[f(x)<y] \cdot\left[f(x)>y^{\prime}\right]$ and thus also for the set $[\|f(x)-f(a)\|<\epsilon]$.

LEMMA 6. In order that $f$ be not approximately continuous at the point $a \in R-R^{*}$ (for $\psi$ relative to $\mathfrak{\mathfrak { O }}$ ), it is necessary and sufficient that there exists either a $y>f(a)$ or a $y^{\prime}<f(a)$, so that the upper density (for $\psi$ relative to $\mathfrak{Q})$ of the set $[f(x) \geqq y]$ or $\left[f(x) \leqq y^{\prime}\right]$ is positive at a.

Necessity. Because of Lemma 5 , there exists either a $y>f(a)$ or a $y^{\prime}<f(a)$, such that the set $[f(x)<y]=A$ or $\left[f(x)>y^{\prime}\right]=A^{\prime}$ has a lower density less than 1 at $a$. But $R-A=[f(x) \geqq y], R-A^{\prime}=\left[f(x) \leqq y^{\prime}\right]$, and, because of Lemma 1 , we have either $\bar{d}(a, R-A, \psi, \mathfrak{Q})>0$ or $\bar{d}\left(a, R-A^{\prime}, \psi, \mathfrak{Q}\right)>0$.

Sufficiency. Using the same notation, we have either $\vec{d}(a, R-A$, $\psi, \mathfrak{Q})>0$ or $\bar{d}\left(a, R-A^{\prime}, \psi, \mathfrak{Q}\right)>0$. Thus because of Lemma 1 , either

$$
\underline{d}(a, A, \psi, \mathfrak{D})<1
$$

or

$$
\underline{d}\left(a, A^{\prime}, \psi, \mathfrak{Q}\right)<1 .
$$

Therefore, because of Lemma $5, f$ is not approximately continuous at $a$.

\footnotetext{
${ }^{15}$ A. Denjoy, loc. cit., p. 165.
}

${ }^{16}$ A. Denjoy, loc. cit., p. 169. 
LEMmA 7. If $|f| \leqq k$ holds, except perhaps on a set of $\psi$-measure zero, and if $f$ is approximately continuous at a, then also $|f(a)| \leqq k$.

Proof. We set $[f(x)>k]=A$; then $\psi(A)=0$. If $f(a)>k$, then, according to Lemma $5, A$ has density 1 at $a$; thus there exists a set $Q \in \mathfrak{Q}_{a}$, such that $\psi(A Q)>0$; therefore we have $\psi(A)>0$, contrary to $\psi(A)=0$. In the same way we see that $f(a)<-k$ is impossible.

Now let the function $f$ be not only $\psi$-measurable in $R$, but also $\psi$-integrable (that is, integrable with respect to the measure $\psi$ ) for all $M \in \mathfrak{M}$ in the sense of Radon and Fréchet $;^{17}$ we set $\phi(M)=(M) \int f d \psi$, where $f$ is integrated with respect to $\psi$ over the set $M \in \mathfrak{M}$. If the $\psi$-measurable function $f$ is $\psi$-bounded (that is, bounded, except perhaps on a set of $\psi$-measure zero), then $f$ is also $\psi$-integrable.

THEOREM 1. Let $\mathfrak{Q}$ be an indefinitely fine system, let $f$ be $\psi$-measurable and $\psi$-bounded ${ }^{18}$ in $R$, and $\phi(M)=(M) \int f d \psi$. If $f$ is approximately continuous at a for $\psi$ relative to $\mathfrak{Q}$, then $\phi$ has the derivative $D(a, \phi, \psi, \mathfrak{Q})=f(a)$ at $a$.

PROOF. Let $Q_{\nu} \in \mathfrak{Q}_{a}$ be a sequence of sets converging to $a$. As we have $a \in R-R^{*}$ according to Lemma 4 , we can assume $\psi\left(Q_{v}\right) \neq 0$. As $f$ is $\psi$-bounded, because of Lemma $7 f(a)$ is finite. For a given $\epsilon>0$, we write: $B=[|f(x)-f(a)|<\epsilon]$. Then we have

$$
\phi\left(Q_{\nu}\right)=\left(Q_{\nu} B\right) \int f d \psi+\left(Q_{\nu}-B\right) \int f d \psi .
$$

Because of $|f(x)-f(a)|<\epsilon$ on $B$, we have

$$
\left(Q_{\nu} B\right) \int f d \psi=\left(f(a)+\theta_{1} \epsilon\right) \cdot \psi\left(Q_{\nu} B\right), \quad\left|\theta_{1}\right| \leqq 1 .
$$

As $f$ is $\psi$-bounded, there is a finite $c$, such that $|f(x)| \leqq c$, except perhaps for a set of $\psi$-measure zero; thus we have

$$
\left(Q_{\nu}-B\right) \int f d \psi=\theta_{2} c \psi\left(Q_{\nu}-B\right), \quad\left|\theta_{2}\right| \leqq 1 .
$$

As $f$ is approximately continuous at $a$, we have $d(a, B, \psi, \mathfrak{Q})=1$, thus $\psi\left(Q_{\nu} B\right) / \psi\left(Q_{\nu}\right) \rightarrow 1$, and therefore

${ }^{17}$ J. Radon, Sitzungsberichte, Akademie der Wissenschaften, Vienna, IIa, vol. 122 (1913), pp. 1324-1332; M. Fréchet, Comptes Rendus de l'Académie des Sciences, Paris, vol. 160 (1915), pp. 839-840; Bulletin de la Scciété Mathématique de France, vol. 43 (1915), pp. 248-265.

18 That the condition, $f$ be $\psi$-bounded, is essential is already shown by an example given by A. Denjoy, loc. cit., p. 173 (for the 1-dimensional case and $\psi$ meaning Lebesguc's linear measure). 


$$
\frac{\psi\left(Q_{\nu}-B\right)}{\psi\left(Q_{\nu}\right)} \rightarrow 0 .
$$

As $\epsilon>0$ is arbitrarily small, both these relations, together with (3), (4), (5) give $\phi\left(Q_{v}\right) / \psi\left(Q_{v}\right) \rightarrow f(a)$.

Theorem 2. In Theorem 1 , the derivative $D(a, \phi, \psi, \mathfrak{Q})$ is regular.

Proof. Let $\left(\left(M_{\nu}\right)\right)$ be a sequence of sets regularly converging to $a$ (for $\psi$ relative to $\mathfrak{Q}$ ); that is, there must exist a sequence $Q_{\nu} \in \mathfrak{Q}_{a}$ converging to $a$, and a $\zeta>0$, such that (1) hold. Because of Lemma 4 , we can assume: $\psi\left(Q_{\nu}\right) \neq 0$, thus according to (1) also $\psi\left(M_{\nu}\right) \neq 0$. Now (1), (6) imply: $\psi\left(M_{\nu}-B\right) / \psi\left(M_{\nu}\right) \rightarrow 0$, and therefore $\psi\left(M_{\nu} B\right) / \psi\left(M_{\nu}\right) \rightarrow 1$. As (3), (4), (5) hold, if we substitute $M_{\nu}$ for $D_{\nu}$, we get as before: $\phi\left(M_{\nu}\right) / \psi\left(M_{\nu}\right) \rightarrow f(a)$.

An inverse of the Theorems 1 and 2 is contained in the following theorem, for which, however, we do not have to assume that $f$ is $\psi$-bounded, but only that it is $\psi$-integrable.

THEOREM 3. Let $\mathfrak{Q}$ be an indefinitely fine system and $\phi(M)=(M) \int f d \psi$. If at the point $a \in R-R^{*}$, the derivative $D(a, \phi, \psi, \mathfrak{Q})$ is regular and equal to $f(a)$, then $f$ is approximately continuous at a (for $\psi$ relative to $\mathfrak{D})$.

Proof. Assume that $f$ is not approximately continuous at $a$ (for $\psi$ relative to $\mathfrak{Q})$. Then, because of Lemma 6 , there exists either a $y>f(a)$ or a $y^{\prime}<f(a)$, such that the upper density (for $\psi$ relative to $\mathfrak{Q}$ ) of the set $[f(x) \geqq y]=C$ or $\left[f(x) \leqq y^{\prime}\right]=C^{\prime}$ is positive at $a$. Suppose, we have the first case (the second case is to be treated in the same way). There exists a sequence of sets $Q_{\nu} \in \mathfrak{\Omega}_{a}$, converging to $a$, so that $\psi\left(C \cdot Q_{\nu}\right) / \psi\left(Q_{\nu}\right) \rightarrow \bar{d}(a, C, \psi, \mathfrak{Q})>0$; thus there exists a $\zeta(a)>0$, so that $\psi\left(C Q_{\nu}\right)>\zeta(a) \cdot \psi\left(Q_{\nu}\right)>0$ for almost all $\nu$, that is, the sequence $\left(\left(C \cdot Q_{\nu}\right)\right)$ converges regularly to $a$ (for $\psi$ relative to $\mathfrak{Q}$ ). Because $f(x) \geqq y$ on $C$ and $y>f(a)$, we therefore have $\phi\left(C Q_{\nu}\right)=\left(C Q_{\nu}\right) \int f d \psi \geqq y \psi\left(C Q_{\nu}\right)$ $>f(a) \psi\left(C Q_{\nu}\right)$ for almost all $\nu$; thus the sequence $\phi\left(C Q_{\nu}\right) / \psi\left(C Q_{\nu}\right)$ cannot converge to $f(a)$.

The Theorems 1, 2, 3, together give the following result:

THEOREM 4. Let $\mathfrak{Q}$ be an indefinitely fine system, let $f$ be $\psi$-measurable and $\psi$-bounded in $R$, and $\phi(M)=(M) \int f d \psi$. In order that at the point $a \in R-R^{*}, \phi$ have the regular derivative $D(a, \phi, \psi, \mathfrak{Q})=f(a)$, it is necessary and sufficient, that $f$ be approximately continuous at a for $\psi$ relative to $\mathfrak{Q}$.

University of New Mexico 